\title{
Choque cardiogénico poscirugía de revascularización de miocardio debido a espasmo coronario
}

\author{
Cardiogenic shock after CABG surgery due to coronary spasm
}

Sebastián Prado-Núñez ${ }^{1 *}$, Jin M. Yamamoto-Kagami ${ }^{1}$, Vicente A. Benites-Zapata ${ }^{1}$, Julio Morón-Castro ${ }^{2}$ y Josías C. Ríos-Ortega ${ }^{2}$

${ }^{1}$ Escuela de Medicina, Universidad Peruana de Ciencias Aplicadas; ${ }^{2}$ Departamento de Cirugía Cardíaca, Instituto Nacional Cardiovascular, INE⿺辶R, EsSalud. Lima, Perú

\section{Presentación del caso}

Paciente mujer de 55 años de edad con historia de infarto agudo de miocardio y desorden cerebrovascular isquémico diagnosticados cuatro años atrás, así como hipertensión, dislipidemia y antecedente de artritis reumatoide de 10 años de evolución tratada con corticoides y metrotexato. Fue admitida por emergencia con diagnóstico de síndrome coronario agudo sin elevación del segmento ST (dolor torácico de un día de evolución e infradesnivel del ST de $2 \mathrm{~mm}$ en derivadas de cara anterior en el electrocardiograma) y elevación de enzimas cardíacas (CPK MB: $45 \mathrm{U} / \mathrm{l}$ ), el cual fue estabilizado en emergencia con terapia médica. La paciente poseía un ecocardiograma 3 meses previos al evento donde poseía una fracción de eyección ventricular izquierda (FEVI) del $45 \%$ y con hipocinesia moderada en cara anterior.

Se le realizó cateterismo cardíaco diagnóstico $24 \mathrm{~h}$ después de su ingreso a emergencia, el cual mostró oclusión severa del $95 \%$ en el ostium coronario izquierdo (Fig. 1) y resto de arterias coronarias sin lesiones angiográficamente significativas; además, se observó circulación colateral heterocoronaria de coronaria derecha a descendente anterior. Dos días después del cateterismo se realizó la cirugía de baipás coronario con el uso de arteria mamaria interna bilateral, colocándose la mamaria izquierda hacia arteria marginal y la derecha a descendente anterior.

En el postoperatorio inmediato ( $2 \mathrm{~h}$ después de la cirugía) la paciente presentó inestabilidad hemodinẩmica franca con índice cardíaco de $2.0 \mathrm{l} / \mathrm{m}^{2}$, pres ón arterial media de $60 \mathrm{mmHg}$ y frecuencia cardíaca de 120 latidos por minuto, a pesar del uso de dobutamina hasta $10 \mathrm{mg} / \mathrm{kg}$ por minuto y noradrenalina hästa $0.3 \mathrm{ug} / \mathrm{kg}$ por minuto, el electrocardiograma môstró infradesnivel del segmento ST de hasta $3 \mathrm{~mm}$ en III, III y aVf, V4, V5, V6 y supradesnivel de $2 \mathrm{~mm}$ en aVR $\mathrm{R}_{\mathrm{D}}^{\mathrm{a}}$ sí como elevación de CPK MB en 15 U/l. La ecocaedipgrafía mostraba una $\mathrm{FEVI}$ del $35 \%$ con hipodinessia severa difusa. Inmediatamente después se colocö́balón de contrapulsación intraaórtico por punción femoral derecha, con lo que se consiguió la estabilización trahsitoria mejorando el índice cardíaco hasta $2.4 \mathrm{l} / \mathrm{m}$ in sin embargo, las alteraciones en el electrocardiogrẩma eran persistentes, por lo que se realizó cateterismo cardíaco de emergencia $4 \mathrm{~h}$ después de la cirugia ånte la sospecha de infarto de miocardio perioperato iōpor oclusión de injertos y con posibilidad de angioplaastia del tronco coronario izquierdo.

Las imágenes de la angiografía coronaria mostraran permeabilidad de ambos baipases; sin embargơ⿱宀 el 


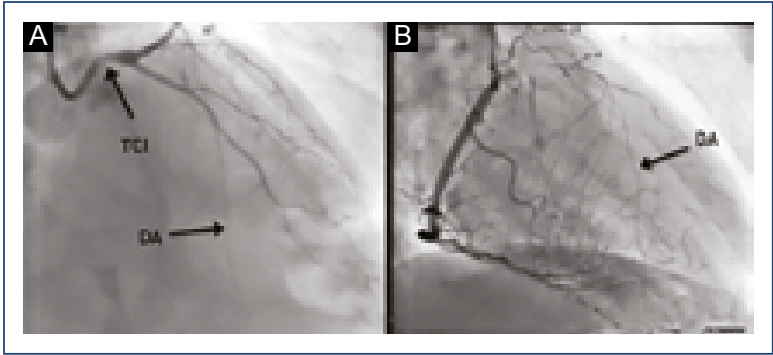

Figura 1. A: coronariografía izquierda que muestra la lesión severa en el ostium del tronco izquierdo y adelgazamiento difuso de la DA. B: coronaria derecha sin obstrucciones y se evidencia la circulación colateral a la DA. CX: arteria circunfleja; DA: arteria descendente anterior; TCl: tronco coronario izquierdo.

flujo de las arterias mamarias era filiforme debido a espasmo severo de ambas arterias y también se observó espasmo severo de las arterias coronarias nativas (Fig. 2 A y B). Con estos hallazgos se suspendió el uso de noradrenalina y se inició terapia vasodilatadora con nitroglicerina guiada por los niveles de presión arterial. A las $48 \mathrm{~h}$ del postoperatorio a la paciente se le retira el balón de contrapulsación y el uso de dobutamina, así como la ventilación mecánica. Se inició terapia farmacológica con verapamilo.

La evolución posterior fue satisfactoria; sin embargo, se realizó un nuevo cateterismo cardíaco tres semanas después de la cirugía, el cual mostró permeabilidad y buen flujo de los baipases y anastomosis coronarias sin lesiones (Fig. $2 \mathrm{C}$ y D). En este tiempo la FEVI era de $40 \%$ y la motilidad se encontró solo hipocinesia leve en región anteroseptal; el resto de los parámetros eran normales. Actualmente la paciente tiene un año de seguimiento con buena evolución clínica, con tratamiento vasodilatador con verapamilo; además de tratamiento antiagregante e hipolipemiante.

\section{Discusión}

El caso presentado es un espasmo coronario postoperatorio que puso a la paciente en shock, pero que se resolvió satisfactoriamente con el uso del balón de contrapulsación aórtico y vasodilatadores. Un estudio analizó el perfil de 115 casos de espasmo coronario perioperatorios y concluyó que la media de edad era 64 años, de los cuales el $84 \%$ eran hombres, y los mayores factores de riesgo eran la hipertensión, historia de angina, ser fumador y tener diabetes mellitus ${ }^{1}$. Cabe resaltar que el uso de doble arteria mamaria se

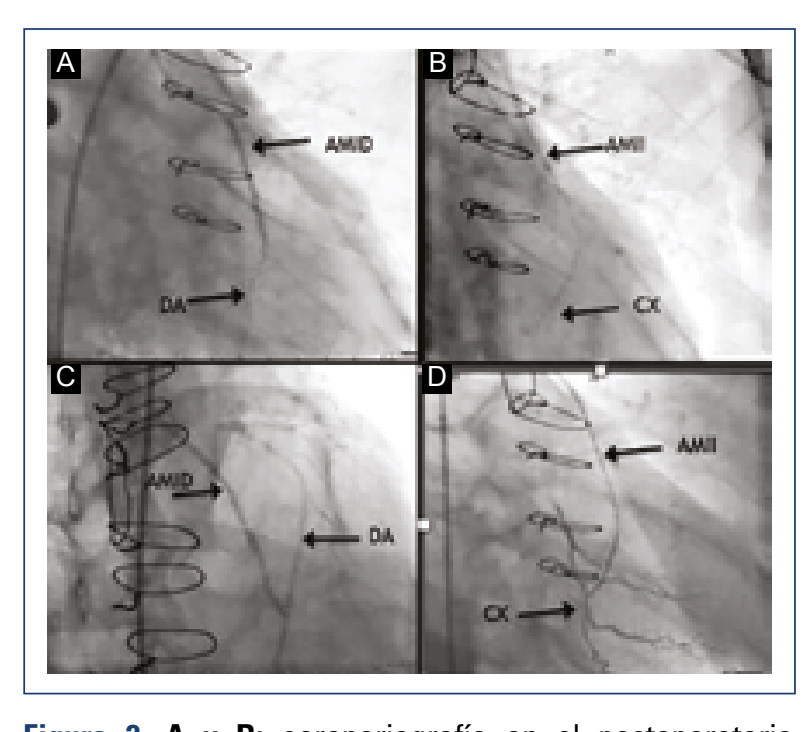

Figura 2. A y B: coronariografía en el postope atorio inmediato, espasmo severo de baipases coronaribs así como de las arterias coronarias nativas. Cy D: coronariografía a la tercera semana del postoperatorio que muestra f fujos adecuados en los injertos mamarios, así como anastom permeables y buenos flujos en DA y CX. AMII: arteria mamaria interna izquierda; AMID: arteria mamaria integrna derecha; CX: arteria circunfleja; DA: arteria descendeênte anterior.

realizó ya que es una paciente menor de 70 años yecon una expectativa de vida mayor a 10 años $^{2}$. La fisiopatología del espasmo coronario perioperatorio nळ ha sido aclarada en detalle. ${ }^{3}$

El espasmo coronario postoperatorio ha sido relacionado con trauma de la arteria coronaria por la mamipulación durante la cirugía, alcalosis, baja temperalura corporal, tubos de drenaje torácicos y factores vasöespásticos de las plaquetas dañadas durante el baippás cardiopulmonar ${ }^{4,5}$. La incidencia durante la prinera hora es de entre el 0.8 al $1.3 \%{ }^{3}$.

Debemos sospechar de espasmo cuando el paciente presenta inestabilidad hemodinámica inexplicable en el postoperatorio. $Y$ cuando se excluyen otras causas de inestabilidad como taponamiento cardíaco y neumotórax a tensión, se debe considerar la angiografía cöro-

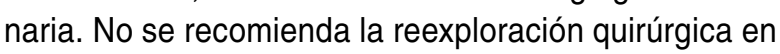
estos casos $^{6}$.

El tratamiento indicado es la inyección intraveno\$a de vasodilatadores como isosorbida, trifosfato de agdenosina o bloqueadores de los canales de calcio. Se reporta que el mejor tratamiento es aplicar inyecciönes intracoronarias de vasodilatadores durante la angiografía coronaria. También es posible poner implantē stents en casos de lesiones focales en espasmos 
refractarios, pero por las complicaciones que trae consigo este tratamiento no es el más recomendado ${ }^{6}$.

En los pacientes con inestabilidad hemodinámica el uso del balón de contrapulsación aórtico puede incrementar el flujo coronario. $Y$ fue este tratamiento junto con los vasodilatadores los que finalmente pudieron ayudar a la paciente a superar la inestabilidad 6 . Así también se reportó el caso de tres pacientes con cuadros similares, de las cuales dos fallecieron y una logro sobrevivir tras el uso de balón de contrapulsación aórtico, llegando a recuperarse totalmente ${ }^{7}$. Generalmente el pronóstico a largo plazo de los pacientes es bueno, la supervivencia a 5 años puede llegar a ser mayor del $94 \% 8$.

A pesar de ser una entidad poco frecuente, es importante que los Servicios de Cirugía la tengan en cuenta como una posible complicación en el periodo postoperatorio. Su diagnóstico temprano y tratamiento adecuado pueden prevenir posibles consecuencias y la muerte del paciente.

\section{Financiación}

La presente comunicación no ha recibido ayudas específicas provenientes de agencias del sector público, sector comercial o entidades sin ánimo de lucro.

\section{Conflicto de intereses}

Los autores declaran no tener ningún conflicto de intereses.

\section{Responsabilidades éticas}

Protección de personas y animales. Los autôres declaran que para esta investigación no se han realizado experimentos en seres humanos ni en animales.

Confidencialidad de los datos. Los autores déclaran que han seguido los protocolos de su centrô de trabajo sobre la publicación de datos de pacientes,

Derecho a la privacidad y consentimiento infiformado. Los autores declaran que en este artícul ॠ no aparecen datos de pacientes.

\section{Bibliografía}

1. Koshiba K, Hoka S. Clinical characteristics of perioperative cokonary spasm: reviews of 115 case reports in Japan. J Anesth. 2001;15(2)-93-9.

2. Kolh P, Windecker S, Alfonso F, Collet JP, Cremer J, Falk V, et a.Task force on myocardial revascularization of the European Society of Cardiology and the European Association for Cardio-Thoracic Surgery. European Ässociation of Percutaneous Cardiovascular Interventions 2014 ESC/EACTS Guidelines on myocardial revascularization: The task force on myocardial revascularization of the European Society of Cardiology (ESC) and the European Association for Cardio-Thoracic Surgery (EACTS). Developede, with the special contribution of the European Association of Percutaneous Oardiovascular Interventions (EAPCI). Eur J Cardiothorac Surg. 2014;46:5\$7-92.

3. Lorusso R, Crudeli E, Lucà F, De Cicco G, Vizzardi E, D'Aloia A, Get al. Refractory spasm of coronary arteries and grafted conduits after isolated coronary artery bypass surgery. Ann Thorac Surg. 2012;93(2):545-51.

4. Schena S, Wildes T, Beardslee MA, Lasala JM, Damiano RJ J!', Lawton JS. Successful management of unremitting spasmof the nongrafted right coronary artery after off-pump coronary artery bypass grafting. J Thorac Cardiovasc Surg. 2007;133(6):1649-50.

5. Pinho T, Almeida J, Garcia M, Pinho P. Coronary artery spasm foltowing aortic valve replacement. Interact Cardiovasc Thorac Surg. 2007;6 3):387-8.

6. Unai S, Hirose H, Cook G, Lee Y, Miura S, Kigawa I, et al. Coronary artery spasm following off-pump coronary artery bypass surgety. Int Heart J. 2014;55(5):451-4.

7. Paterson HS, Jones MW, Baird DK, Hughes CF. Lethal postoperative coronary artery spasm. Ann Thorac Surg. 1998;65(5):1571-3.

8. Ong $P$, Athanasiadis A, Borguiya G, Voehringer M, Sechtem U. 3Qjears follow-up of patients with coronary artery spasm as cause of adutecoronary síndrome: the CASPAR (coronary artery spasm in patients with acute coronary síndrome) study follow-up. J Am Coll Cardiol. 2011;57(2\$147. 